\title{
Efficient forecasting model technique for river stream flow in tropical environment
}

\begin{abstract}
Monthly stream flow forecasting can provide crucial information on hydrological applications including water resource management and flood mitigation systems. In this statistical study, time series and artificial intelligence methods were evaluated according to implementation of each time-series technique to find an effective tool for stream flow prediction in flood forecasting. This paper explores the application of water level, rainfall data and input time series into three different models; linear regression (LR), auto-regressive integrated moving average (ARIMA) and artificial neural networks (ANN). The performances of the models were compared based on the maximum coefficient of determination $\left(\mathrm{R}^{2}\right)$ and minimum root means square error (RMSE). Based on the results the ANN model presents the most accurate measurement, with the $\mathrm{R}^{2}$ value of 0.868 and $18 \%$ RMSE. The present study suggests that ANN is the best model due to its ability to recognise times series patterns and to understand non-linear relationships.
\end{abstract}

Keyword: Linear regression; ARIMA; Artificial neural networks; Flood forecasting 